\title{
ADAPTACIÓN DEL SECTOR VITIVINÍCOLA AL CAMBIO CLIMÁTICO EN LA D.O. CARIÑENA
}

\author{
$\underline{\text { Raúl Compés }}^{a^{*}}$ y Marta Mompeón ${ }^{b}$
}

a Centro Internacional de Altos Estudios Agronómicos Mediterráneos (CIHEAM) (Zaragoza, raul.compes@iamz.ciheam.org)

${ }^{b}$ Universitat Politècnica de València (Valencia - España, mmompeon@gmail.com)

\section{Resumen}

La aceleración del cambio climático amenaza gravemente a la producción agrícola, y uno de los sectores más afectados es el vitivinícola. Aunque los escenarios futuros son inciertos, todo apunta a que la situación climatológica en las regiones mediterráneas va a dificultar aún más el cultivo de la vid. Sin embargo, debido a que el calentamiento no es homogéneo y cada zona vitivinícola cuenta con un microclima propio, los impactos y las estrategias relevantes dependen de las características locales.

Esta comunicación se centra en el cambio climático en la Denominación de Origen Protegida (D.O.P) Cariñena. Los objetivos específicos son: a) conocer de qué manera el cambio climático está afectando a la actividad vitícola en la zona, b) estimar la intensidad con qué lo hará en los próximos años y c) estudiar y comparar los costes de inversión de las principales medidas de adaptación en el viñedo. Los resultados confirman un empeoramiento de las condiciones actuales, una pérdida de potencial vitivinícola a medio plazo y la conveniencia de acometer medidas de adaptación en el corto plazo, que son menos costosas que las de largo plazo.

Palabras clave: DOP Cariñena, cambio climático, costes adaptación

\section{Introducción}

La aceleración del cambio climático en las últimas décadas es una realidad que amenaza gravemente a los distintos ecosistemas, siendo la agricultura uno de los sectores más vulnerables (Iglesias et al., 2011). La vid, al exigir unas condiciones ambientales muy específicas para producir en la cantidad y calidad comercialmente viable, es un cultivo especialmente sensible (Salazar y Melgarejo, 2005).

Aunque el futuro es incierto, los análisis de escenarios apuntan a que la situación climatológica en las actuales regiones mediterráneas va a ser cada vez más difícil (MedECC, 2019). Sin embargo, debido a que el calentamiento no es homogéneo y cada zona vitivinícola cuenta con un microclima propio, es necesario realizar los estudios de cambio climático a la menor escala posible. Solo así es posible identificar con precisión los principales impactos y valorar con más criterio las medidas de adaptación.

Esta Comunicación se centra en el análisis de los efectos del cambio climático en la Denominación de Origen Protegida (D.O.P) Cariñena, la D.O. vitivinícola más extensa y antigua de Aragón. Situada en pleno valle del Ebro, presenta un clima mediterráneo continental, cuyos rasgos más sobresalientes son la escasez de precipitaciones junto con los elevados contrastes térmicos anuales y diarios. Sus viñedos se distribuyen entre los 400 y 800 metros de altitud, por lo que existen notables diferencias en las características climáticas de las distintas subzonas existentes. Los objetivos específicos de la Comunicación son: a) conocer de qué manera el cambio climático está afectando a la actividad vitícola en la zona, b) estimar la intensidad con qué lo hará en los próximos años y c) estudiar y comparar los costes de las estrategias de adaptación más importantes. Los datos meteorológicos proceden de la AEMET, y los costes de proveedores de servicios auxiliares de referencia en la zona.

\section{Cambios climatológicos recientes en la zona de estudio}

Los registros climáticos disponibles en la AEMET permiten caracterizar la situación climática de la comarca. Los datos se refieren exactamente a la localidad de Cariñena, que se sitúa a una altitud de $591 \mathrm{~m}$. En el período analizado se han producido cambios sustanciales en los parámetros climáticos más importantes. En primer lugar, la temperatura media anual ha seguido una tendencia ascendente, llegando a incrementarse $0,8^{\circ} \mathrm{C}$ en el periodo 1990-2019 (Figura 1). 
Figura 1. Evolución de la temperatura media anual en Cariñena (1990-2019)

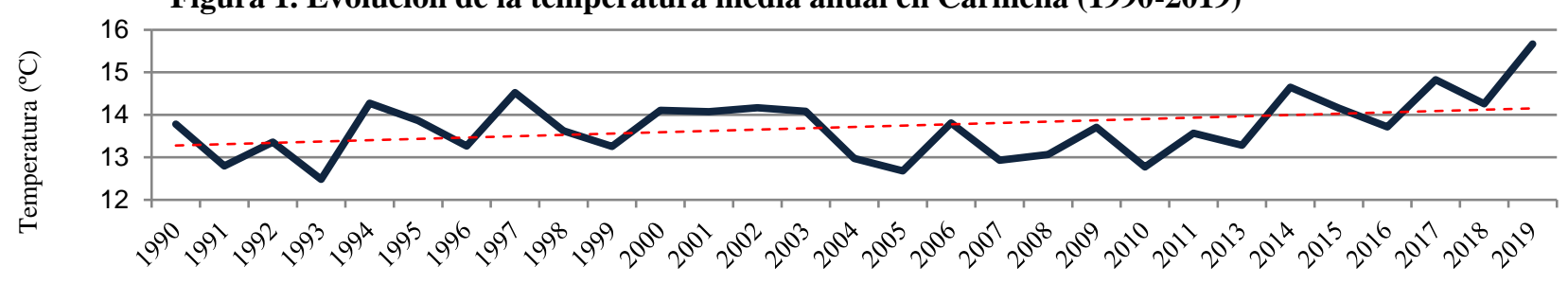

Año

T $\mathrm{T}^{\mathrm{a}}$ Media Anual $\quad$ - - - - Lineal (Ta Media Anual)

Fuente: Elaboración propia a partir datos de AEMET

En segundo lugar, en materia de precipitaciones, además de una gran irregularidad, se ha producido un notable descenso, mucho más acusado en el primer periodo analizado (1970-1989), reduciéndose prácticamente a la mitad (Figura 2). Finalmente, es importante destacar que los episodios de tormentas con pedrisco, los cuales pueden causar importantes daños directos en el cultivo, han aumentado de forma notable, incrementándose aproximadamente en 1,5 días al año entre 1970 y 2019.

Figura 2. Variaciones registradas en las precipitaciones anuales acumuladas (1970-1989)

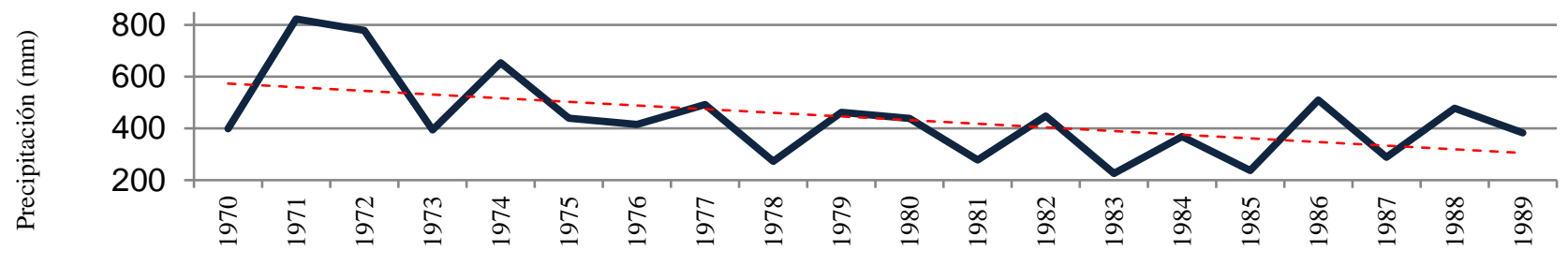

Año

$\longrightarrow$ Precipitación anual - - - - Lineal (Precipitación anual)

Fuente: Elaboración propia a partir datos de AEMET

3. Escenarios futuros y evolución del potencial vitícola

Si bien los datos históricos son necesarios para conocer las tendencias, las decisiones de adaptación se deben hacer teniendo en cuenta las previsiones climáticas. Para estimarlas se ha utilizado como periodo de referencia 1971-2000 y, como período futuro, 2021-2050. Los datos proceden del visor de escenarios de cambio climático (http://escenarios.adaptecca.es), desarrollado en el marco del PNACC (Plan Nacional de Adaptación al Cambio Climático), teniendo en cuenta dos escenarios distintos: uno de emisiones medias (RCP 4.5) y otro de emisiones altas (RCP 8.5).

Con los datos obtenidos, es posible hacer una previsión de la evolución del potencial vitícola en la zona, calculado éste a partir de los índices del Sistema de Clasificación Climática Multicriterio Geovitícola (CCMG) de Tonietto y Carbonneau (2004) (Tabla 1).

Tabla 1. Valores de IH, IF, IS y clasificación climática para los diferentes períodos y escenarios estudiados

\begin{tabular}{|c|c|c|c|}
\hline & $\begin{array}{c}\mathrm{IH} \\
\text { (Índice de } \\
\text { Huglin) }\end{array}$ & $\begin{array}{c}\text { IF } \\
\text { (Índice de noches } \\
\text { frías) }\end{array}$ & $\begin{array}{c}\text { IS } \\
\text { (Índice de sequía) }\end{array}$ \\
\hline $\begin{array}{l}\text { Periodo histórico } \\
\text { (1971-2000) }\end{array}$ & $\begin{array}{c}\text { 2198,99: } \mathrm{IH}+1: \\
\text { Templado } \\
\text { caluroso }\end{array}$ & $\begin{array}{l}\text { 12,03: IF+1: } \\
\text { Noches frías }\end{array}$ & $\begin{array}{c}\text {-59,38: IS+1: } \\
\text { Sequía moderada }\end{array}$ \\
\hline $\begin{array}{c}\text { Escenario futuro RCP } \\
4.5(2021-2050)\end{array}$ & $\begin{array}{l}\text { 2612,84: } \mathrm{IH}+2: \\
\text { Caluroso }\end{array}$ & $\begin{array}{c}\text { 15,02: IF-1: } \\
\text { Noches templadas }\end{array}$ & $\begin{array}{l}\text {-102,92: IS+2: } \\
\text { Sequía fuerte }\end{array}$ \\
\hline
\end{tabular}




\begin{tabular}{|c|c|c|c|}
\hline $\begin{array}{c}\text { Escenario futuro RCP } \\
8.5(2021-2050)\end{array}$ & $\begin{array}{c}\text { 2681,52: IH+2: } \\
\text { Caluroso }\end{array}$ & $\begin{array}{c}15,73: \text { IF-1: } \\
\text { Noches templadas }\end{array}$ & $\begin{array}{c}-108,03: \text { IS+2: } \\
\text { Sequía fuerte }\end{array}$ \\
\hline
\end{tabular}

Fuente: Elaboración propia a partir de datos de http://escenarios.adaptecca.es

Con respecto al Índice de Huglin (IH), aumenta en los dos escenarios futuros, en el caso del RCP 4.5, un $10 \%$, y en el RCP 8.5 , un $16 \%$, por lo que la zona pasa a clasificarse como IH+2: Clima caluroso (2400$\left.3000^{\circ} \mathrm{C}\right)$, caracterizado por un potencial que supera las necesidades heliotérmicas necesarias para que madure cualquier variedad, incluso las más tardías, existiendo riesgos de estrés térmico.

Con respecto al Índice de Frescor Nocturno (IF), pasa de IF+1 - "De noches frías"-, a IF-1 -“De noches templadas" - en los dos escenarios futuros, provocando una menor oscilación térmica diaria durante el periodo de maduración, lo cual puede afectar negativamente al color de la baya y al potencial aromático.

Respecto al Índice de Sequía (IS), durante el periodo de referencia disminuyó alrededor de un 490\%, clasificándose como IS+1 - "De sequía moderada"-. En los dos escenarios futuros el valor de este índice sigue descendiendo, siendo en el caso del RCP 8.5 más notable la caída, un 102\%, por lo que en ambos la zona pasa a clasificarse como IS+2 - "De sequía fuerte $(>-100 \mathrm{~mm})$ "-, donde en la mayoría de los casos el riego es una práctica imprescindible debido al frecuente estrés hídrico existente.

Por tanto, según este análisis, la D.O.P Cariñena se enfrenta a un empeoramiento de las condiciones climatológicas con respecto al periodo de referencia, con el resultado de pasar a clasificarse -en los dos escenarios analizados- como zona de bajo potencial climático para la viticultura, con consecuencias negativas para el cultivo y con alteraciones en la tipicidad de los vinos allí producidos.

\section{Análisis económico de medidas de adaptación con costes de inversión}

Teniendo en cuenta estas previsiones negativas, y con el fin de mantener la viabilidad del cultivo en la comarca, es necesario realizar un estudio de alternativas de adaptación. Según la literatura existente, hay una correlación positiva entre la gravedad de las consecuencias negativas del cambio climático en una zona, el plazo de tiempo, el tipo de medidas a implementar y su coste y dificultad de aplicación (Iglesias et al., 2011). De esta forma, hay que distinguir entre medidas a corto plazo, que se suponen de más fácil implantación, aunque con efectos limitados ante grandes cambios, y medidas a largo plazo que requieren más inversión y tiempo de implantación.

Las adaptaciones a corto plazo pueden ser consideradas como la primera estrategia de protección contra el cambio climático. En su mayoría, implican cambios en las prácticas enológicas a través de los avances tecnológicos (Lobell et al., 2006), con el objetivo de mejorar la calidad del vino producido. A corto y medio plazo, las medidas en viticultura se basan en el cambio de variedad, la puesta en riego y la gestión del viñedo, que implican más esfuerzo, pero a su vez pueden mejorar la adaptación ante cambios más pronunciados. Por último, las medidas a largo plazo suponen replantar la viña, cambiando todo el material vegetal buscando una mejor aclimatación de las cepas, y pueden llegar hasta su arranque y desplazamiento a una localización nueva, lo cual supone importantes esfuerzos económicos, asociados además a grandes riesgos. Para contrastar esta hipótesis se han estimado los costes de las medidas que afectan a la viticultura y conllevan inversiones en activos fijos. Evidentemente estos cambios afectan a la producción -en cantidad y calidad- y, por tanto a los ingresos y costes de explotación del cultivo, pero su análisis queda fuera de esta Comunicación.

Como primera medida de adaptación se considera la puesta en riego de un viñedo típico de la zona en secano, mediante la implantación un sistema de riego localizado por goteo con fertirrigación. El regadío es un factor clave en la adaptación del viñedo, pero supone una incertidumbre por la ausencia de garantías de disponibilidad de agua y por la competencia con otros cultivos en la zona. Se espera un incremento de la demanda de agua de riego, lo que incrementa la presión por unos recursos hídricos que comienzan a escasear, cuestionando la sostenibilidad de esta medida de adaptación. El coste total medio para poner en riego una hectárea en la zona es de $8.528,1 € /$ ha.

La segunda medida de adaptación es el cambio de variedad, seleccionando un material vegetal mejor adaptado a las condiciones climáticas previstas. Sin tener que arrancar la viña, la forma más rápida es mediante el reinjerto de cepas ya existentes, manteniendo el portainjerto sobre el que se establece la variedad, perdiendo únicamente la producción de ese año. Las variedades elegidas por su mejor adaptación, debido a su maduración tardía, tolerancia a la sequía y gran rusticidad son: Garnacha tinta -la más utilizada en la D.O.P.-, Cariñena o Mazuela, y Vidadillo o Crespiello, una variedad autóctona muy antigua y casi olvidada en la actualidad. El coste total de cambio de variedad es $3.751 € /$ ha, teniendo en cuenta un éxito del injerto del $70 \%$. 
Si la evolución futura se escora hacia el escenario RCP 8.5 quizá sea necesario ir hacia la replantación, la cual consiste en arrancar una viña y sustituirla por otra, cambiando en este caso tanto la variedad como el portainjerto, por lo que se elige un material vegetal nuevo, mejor adaptado al clima futuro previsto. En el estudio realizado se ha seleccionado $110 \mathrm{R}$ como portainjerto de la nueva plantación, por ser tolerante a la sequía, estar muy adaptado a zonas cálidas y retrasar la maduración de los cultivares injertados sobre él (Salazar y Melgarejo, 2005). Los costes totales de este medida para el caso estudiado son de 16.376,62€/ha.

La medida más drástica de adaptación es la deslocalización del viñedo. En este análisis se considera un desplazamiento en altitud dentro del territorio de la propia D.O.P., hacia la Sierra de Algairén, con el objetivo de ascender en altitud para ubicar el cultivo en zonas más frías. En concreto se simula una nueva plantación a 860 metros de altitud, entre los municipios de Encinacorba y Paniza, una de las zonas más altas de la D.O.P. Con ello la precipitación media anual puede aumentar hasta un rango medio de 475-500 mm y, además, se consigue suavizar las temperaturas más extremas que caracterizan los meses más calurosos. Esta medida conlleva unos costes totales de $28.714 € / \mathrm{ha}^{77}$, estimando que una hectárea en la nueva localización tiene un precio aproximado de 7.500€.

Por tanto, comparando las cuatro medidas de adaptación con costes de inversión, la más barata es el sobreinjerto (3.751€/ha), seguida -en orden de menor a mayor- por la instalación de riego $(8.528,1 € /$ ha $)$, la replantación del viñedo $(16.376,62 € /$ ha) y la deslocalización de la viña $(28.714 € /$ ha); lo que confirman los trabajos realizados en otras zonas (Iglesias et al., 2011).

\section{Conclusiones}

En la zona de la D.O.P. Cariñena se viene produciendo durante las últimas décadas una reducción sustancial de las precipitaciones, un aumento de las temperaturas y un incremento de la intensidad y frecuencia de fenómenos extremos, lo que encaja con el fenómeno de cambio climático. Los pronósticos para las próximas décadas auguran un empeoramiento de las condiciones de cultivo en cualquiera de los escenarios que parecen cada vez más probables (RCP 8.5 y RCP 4.5). Esto supone que la D.O.P. podría pasar a clasificarse como una zona de bajo potencial climático, lo que pondría en peligro la viabilidad del cultivo en las zonas más frágiles.

La adopción de medidas de adaptación va a ser, por tanto, una necesidad cada vez mayor en las zonas más vulnerables. Si bien en cada caso habrá que realizar un análisis técnico y económico específico, los resultados de este trabajo, a partir de un estudio de casos, corroboran la hipótesis de la literatura. Los costes de inversión, uno de los parámetros básicos en la elección de las medidas de adaptación, y que guardan una relación muy estrecha con la intensidad del problema, son menores en el caso de reinjerto con variedades más resistentes, por lo que debería ser una de las primeras medidas de adaptación. En términos estrictamente económicos, la replantación del viñedo y la deslocalización solo se justifican en caso de especial agravamiento del problema, en cuyo caso pueden ser imprescindibles.

\section{Bibliografía}

Iglesias, A., Quiroga Gómez, S. y Sotés Ruiz, V. (2011). "La agricultura española y el cambio climático" Economistas, p. 127, 19-26.

Llobell, D.; Field, C.; Cahill, K. y Bonfils, C. (2006). "Impacts of future climate change on California perennial crop yields: Model projections with climate and crop uncertainties". Agric For Meteorol. 141(24); p. 208-218.

Medecc (2019). "Risks associated to climate and environmental changes in the Mediterranean region". The British Journal of Psychiatry, 479, p. 1009-1010.

Tonietto, J., y Carbonneau, A. (2004). “A Multicriteria Climatic Classification System for grape-growing regions worldwide" Agricultural Forest Meteorology 124(1-2), p. 81-97.

Salazar, D. M. y Melgarejo, P. (2005). "Viticultura: Técnicas de cultivo de la vid, calidad de la uva y atributos de los vinos. Mundi-Prensa Libros S.A. Madrid. p. 325.

77 A este coste habría que sumar la necesidad de contar con una autorización y el arranque de la viña anterior. 